\title{
A confined flare above filaments
}

\author{
K. Dalmasse ${ }^{1}$, R. Chandra ${ }^{2}$, B. Schmieder ${ }^{1}$, and G. Aulanier ${ }^{1}$ \\ ${ }^{1}$ LESIA, Observatoire de Paris, CNRS, UMPC, Univ. Paris Diderot, \\ 5 place Jules Janssen, 92190 Meudon, France \\ email: kevin.dalmasse@obspm.fr \\ ${ }^{2}$ Dept. of Physics, DSB Campus, Kumaun University, Nainital- 263 002, India
}

\begin{abstract}
We present the dynamics of two filaments and a C-class flare observed in NOAA 11589 on 2012 October 16. We used the multi-wavelength high-resolution data from SDO, as well as THEMIS and ARIES ground-based observations. The observations show that the filaments are progressively converging towards each other without merging. We find that the filaments have opposite chirality which may have prevented them from merging. On October 16, a C3.3 class flare occurred without the eruption of the filaments. According to the standard solar flare model, after the reconnection, post-flare loops form below the erupting filaments whether the eruption fails or not. However, the observations show the formation of post-flare loops above the filaments, which is not consistent with the standard flare model. We analyze the topology of the active region's magnetic field by computing the quasi-separatrix layers (QSLs) using a linear force-free field extrapolation. We find a good agreement between the photospheric footprints of the QSLs and the flare ribbons. We discuss how slipping or slip-running reconnection at the QSLs may explain the observed dynamics.
\end{abstract}

Keywords. Filaments, flare, MHD

\section{Introduction}

Filaments are dark, elongated structures consisting of chromospheric plasma embedded in the much hotter corona (van Ballegooijen \& Martens 1989; Chae et al. 2001). They are cool $(\approx 8000 \mathrm{~K})$, dense material confined in highly stressed magnetic fields overlying polarity inversion lines (PILs; Aulanier \& Démoulin 1998; Schmieder et al. 2006). In the standard picture, the magnetic structure of filaments is formed through shearing motions along PILs and/or, through magnetic flux cancellation due to converging motions of opposite magnetic polarities towards the PILs (van Ballegooijen \& Martens 1989; Antiochos et al. 1994).

Eventually, filaments may become unstable (see Moore et al. 2001; Martens \& Zwaan 2001). According to the standard solar flare model (hereafter, CSHKP model), the instability of the filament may lead to two different types of flares, namely, eruptive or confined flares (see review by Shibata \& Magara 2011). Eruptive flares correspond to cases for which the filament erupts, leading to the formation of a coronal mass ejection (CME). On the other hand, confined flares are sometimes associated with cases for which the eruption of the filament fails (e.g., Török \& Kliem 2005). Confined flares also comprise flares induced by magnetic reconnection of different magnetic flux tubes, or magnetic coronal loops, for which no filament is present (e.g., Berlicki et al. 2004; Chandra et al. 2006). In the context of flares involving the presence of a filament, the CSHKP model predicts for both eruptive and confined flares, that the flare will be associated with two flare ribbons, and with the formation of hot post-flare loops below the erupting filament, regardless of whether it is a successful or failed eruption. 
In this study, we present the evolution of two filaments and a confined flare observed in NOAA 11589, which cannot be explained by the CSHKP model. We propose an alternative flare scenario which accounts for the observed flare signatures and filaments evolution during the flare.

\section{Observations}

Our study was performed by combining observations from the Solar Dynamic Observatory (SDO) satellite, the french Télescope Héliographique pour l'Etude du Magnétisme et des Instabilités Solaires (THEMIS), and an indian telescope of the Aryabhatta Research Institute of observational Sciences (ARIES).

NOAA 11589 appeared on 2012 October 10 at the heliographic coordinates N13 E61. The AR quickly developed into two decaying magnetic polarities (see Fig. 1a). During its on-disk passage, the AR was associated with large-scale magnetic flux cancellation, and a few localized magnetic flux emergence events.

The flux cancellation in the internal part of the AR led to the formation of two filaments of opposite chirality which eventually converged. However, the filaments did not merge probably due to their axial field being oriented in opposite direction along the PIL (e.g., Schmieder et al. 2004; DeVore et al. 2005).

The AR also presented some recurring and localized magnetic flux emergence associated with Ellerman bombs (EBs) in its northern part (as in Pariat et al. 2004).

On October 16, the AR produced a confined C3.3 class flare which started around 16:00 UT and ended around 16:39 UT. A first analysis of the flare signatures with AIA $1600 \AA$ and AIA $171 \AA$ channels seem to be in agreement with the CSHKP model: apparently presenting two flare ribbons, and the formation of hot post-flare loops.

However, a careful analysis of the EUV data from the AIA $171 \AA$ channel reveals that the flare did not lead to the eruption of any of the filaments. It also shows a striking result: the post-flare loops were formed above the filaments contrary to what is expected from the CSHKP model, and the filaments were not disturbed by the flare.

\section{Analysis}

\subsection{Magnetic field extrapolation}

To understand and explain the evolution of the filaments during the flare, we study the magnetic topology of the AR by means of an LFFF extrapolation $(\vec{\nabla} \times \vec{B}=\alpha \vec{B}$, with $\alpha$ being the force-free parameter) to identify the key sites for the development of magnetic reconnection that led to the flare.

We only considered the global magnetic field of the AR because (i) the filaments were in plage regions where the magnetic field is weak, and thus, the currents are not well measured, and (ii) the filaments did not seem to play any role in the flare.

The extrapolations were performed using the fast Fourier transform method (Alissandrakis 1981) with a non-uniform grid of $1024^{2} \times 351$ points covering a domain of $700^{2} \times 2000 \mathrm{Mm}^{3}$. Within the set of performed extrapolations, we kept the solution $\alpha=7 \times 10^{-3} \mathrm{Mm}^{-1}$ because it gave the best match with the northern loops of the AR (Fig. 1a), i.e., the region where the flare was initiated.

\subsection{Topological analysis}

The topology is then analyzed by computing the quasi-separatrix layers (QSLs; e.g., Démoulin et al. 1996). QSLs are thin 3D volumes of very sharp gradients of the magnetic field connectivity. QSLs are preferential sites for the build-up of strong and thin 

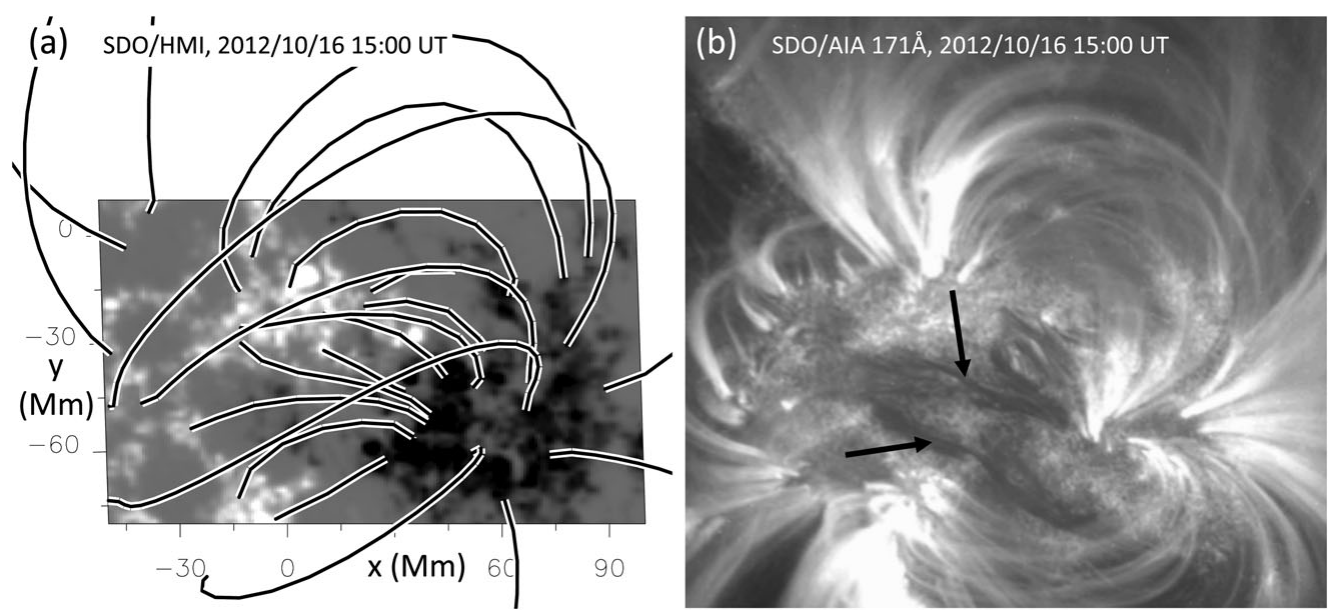

Figure 1. Central part of NOAA 11589. (a) Photospheric vertical magnetic field, $B_{z}$, in greyscale overplotted with selected magnetic field lines from the extrapolation (black lines). (b) AIA171 image showing some of the AR loops and the two observed filaments highlighted by black arrows.

current layers, and for the development of magnetic reconnection at these current layers (see review by Démoulin 2006). As separatrices, QSLs are preferential sites for particle acceleration (Aulanier et al. 2006). Many observational studies have thus successfully compared and associated the photospheric footprints of QSLs to flare ribbons providing indirect evidence of magnetic reconnection as the triggering mechanism of solar eruptive events (e.g., Démoulin et al. 1996; Mandrini et al. 1997; Schmieder et al. 1997). The photospheric mapping of QSLs can be obtained by computing the squashing degree, $Q$ (Titov et al. 2002). QSLs are thus identified as 3D regions of strong $Q$-values $(Q \gg 2)$.

We computed the squashing degree for our LFFF extrapolation using "method 3" of Pariat \& Démoulin (2012). Fig. 2(a) displays the photospheric mapping of QSLs for the same field of view as Fig. 1(a) by representing $\log _{10} Q$ at the photosphere. By plotting magnetic field lines over the photospheric $Q$-map, we identified two double-C shape QSLs, $Q_{1,2}$, similar to Aulanier et al. (2005), and a circular-like one (overlaid with a white circle), $Q_{3}$, similar to Masson et al. (2009). We find a few discrepancies between the QSLs footprints, $Q_{i}$, and the three flare ribbons of Fig. 2(b), $R_{i}$. This is due to the assumptions made by extrapolating the AR's magnetic field in LFFF, which do not model the highly-stressed filament magnetic fields, and which results in local modifications of the magnetic connectivity that slightly modifies the location and shape of the QSLs in our extrapolation. Nevertheless, there is a good qualitative agreement between the QSLs footprints and the flare ribbons (Fig. 2).

\section{Conclusion}

From the previous analysis, it is clear that the magnetic field of AR 11589 presents a complex topology formed by three entangled QSLs. Such a complex topology was favorable to the build-up of electric current layers and to the development of magnetic reconnection at any of these QSLs. The flare might thus have been the result of the stress of, at least, one of the QSLs eventually triggering magnetic reconnection at all QSLs.

Analyzing the AIA and HMI data prior to, and after the flare, we found signatures of localized, recurring magnetic flux emergence in the northern part of the AR - in the region below $Q_{1}$, i.e., between the western part of $Q_{1, \text { curv }}$ and the southern part of $Q_{1, \text { arc }}$. 

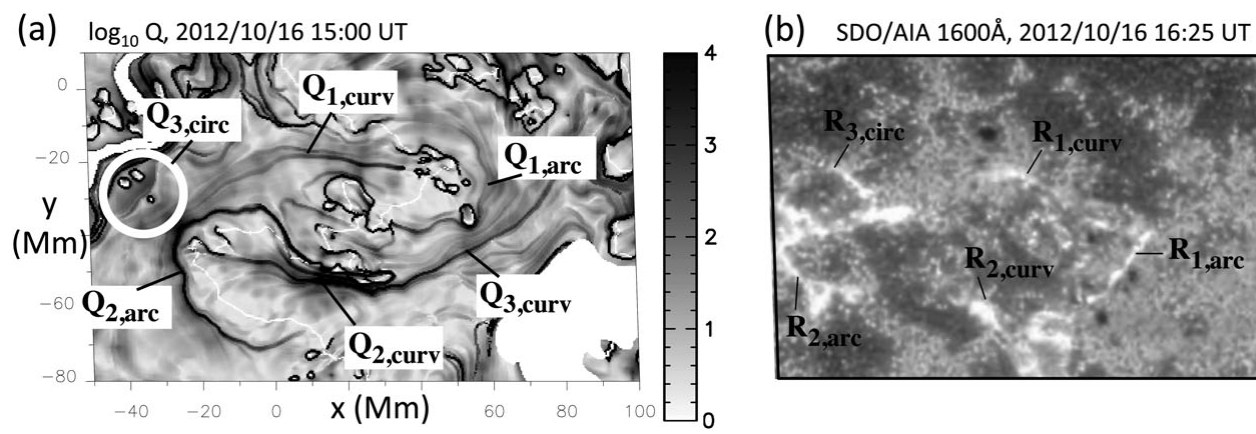

Figure 2. Central part of NOAA 11589. (a) Photospheric mapping of $\log _{10} Q$ displaying the photospheric footprints of QSLs at 15:00 UT. (b) Flare ribbons at 16:25 UT. The footprints of three QSLs, labelled $Q_{i}$, are identified with the three flare ribbons labelled $R_{i}$.

Consequently, we propose that this episodic flux emergence was the driver of the C3.3 class flare: this continuous magnetic flux emergence may have stressed the magnetic field of $Q_{1}$, resulting in the formation of a strong thin current layer, at least, within this QSL. Eventually, this can trigger slipping or slip-running reconnection at $Q_{1}$ (see Aulanier et al. 2006), which, in turn, can trigger magnetic reconnection at all the other intersecting QSLs, $Q_{2}$ and $Q_{3}$. This would have induced particle acceleration at all QSLs (e.g., Masson et al. 2009), and hence, the formation of a complex distribution of flare ribbons (such as shown by Fig. 2b). Since both filaments were located below the QSLs involved in the flare mechanism, our scenario naturally accounts for the development of post-flare loops above these non-erupting filaments.

\section{References}

Alissandrakis, C. E. 1981, A\&SA, 100, 197

Antiochos, S. K., Dahlburg, R. B., \& Klimchuk, J. A. 1994, ApJ, 420, L41

Aulanier, G. \& Démoulin, P. 1998, A\&A, 329, 1125

Aulanier, G., Pariat, E., \& Démoulin, P. 2005, A\& $A$, 444, 961

Aulanier, G., Pariat, E., Démoulin, P., \& DeVore, C. R. 2006, Solar Phys., 238, 347

Berlicki, A., Schmieder, B., Vilmer, N., Aulanier, G., \& Del Zanna, G. 2004, A\&A, 423, 1119

Chae, J., Wang, H., Qiu, J., Goode, P. R., Strous, L., \& Yun, H. S. 2001, ApJ, 560, 476

Chandra, R. et al. 2006, Solar Phys., 239, 239

Démoulin, P. 2006, Adv. Sp. Res., 37, 1269

Démoulin, P., Henoux, J. C., Priest, E. R., \& Mandrini, C. H. 1996, A\&̈A, 308, 643

DeVore, C. R., Antiochos, S. K., \& Aulanier, G. 2005, ApJ, 629, 1122

Mandrini, C. H. et al. 1997, Solar Phys., 174, 229

Martens, P. C. \& Zwaan, C. 2001, ApJ, 558, 872

Masson, S., Pariat, E., Aulanier, G., \& Schrijver, C. J. 2009, ApJ, 700, 559

Moore, R. L., Sterling, A. C., Hudson, H. S., \& Lemen, J. R. 2001, ApJ, 552, 833

Pariat, E., Aulanier, G. et al. 2004, ApJ, 614, 1099

Pariat, E. \& Démoulin, P. 2012, A\& A, 541, A78

Schmieder, B., Aulanier, G., Démoulin, P. et al. 1997, A\&A, 325, 1213

Schmieder, B., Aulanier, G., Mein, P., \& López Ariste, A. 2006, Solar Phys., 238, 245

Schmieder, B., Mein, N. et al. 2004, Solar Phys., 223, 119

Shibata, K. \& Magara, T. 2011, Living Reviews in Solar Phys., 8, 6

Titov, V. S., Hornig, G., \& Démoulin, P. 2002, Journal of Geophysical Research, 107, 1164

Török, T. \& Kliem, B. 2005, ApJ, 630, L97

van Ballegooijen, A. A. \& Martens, P. C. H.. 1989, ApJ, 343, 971 